\title{
Wavelet analysis of cerebral oxygenation oscillations in the screening of Moyamoya disease $^{1}$
}

\author{
Ying $\mathrm{He}^{\mathrm{b}}$, Pengjun Jiang ${ }^{\mathrm{b}}$, Shanshan Han ${ }^{\mathrm{a}}$, Rong Wang ${ }^{\mathrm{b}}$, Yue $\mathrm{Li}^{\mathrm{c}}$, Yichao Teng ${ }^{\mathrm{c}}$ and Tianxin \\ $\mathrm{Gao}^{\mathrm{a}, *}$ \\ ${ }^{\mathrm{a}}$ School of Life Science, Beijing Institute of Technology, Beijing 100081, P.R. China \\ ${ }^{\mathrm{b}}$ Beijing Tian Tan Hospital, Capital Medical University, Beijing 100050, P.R. China \\ ${ }^{\mathrm{c}}$ School of Medicine, Tsinghua University, Beijing 100084, P.R. China
}

\begin{abstract}
Near-infrared spectroscopy (NIRS) was used to investigate the cerebral oxygenation of Moyamoya and healthy subjects. Continuous recordings of NIRS signals for $20 \mathrm{~min}(20 \mathrm{~min}$ signals) were obtained from 17 healthy subjects (age: 37.4 \pm 11.3 ) and 17 Moyamoya subjects (age: 40.1 \pm 11.2 ). Spectral analysis based on wavelet transformation identified five frequency intervals (I, $0.0095 \mathrm{~Hz}$ to $0.02 \mathrm{~Hz}$; II, $0.02 \mathrm{~Hz}$ to $0.06 \mathrm{~Hz}$; III, $0.06 \mathrm{~Hz}$ to $0.15 \mathrm{~Hz}$; IV, $0.15 \mathrm{~Hz}$ to $0.40 \mathrm{~Hz}$; and V, $0.40 \mathrm{~Hz}$ to $2.00 \mathrm{~Hz}$ ) in the 20 min signals and three frequency intervals (III, $0.06 \mathrm{~Hz}$ to $0.15 \mathrm{~Hz}$; IV, $0.15 \mathrm{~Hz}$ to $0.40 \mathrm{~Hz}$; and $\mathrm{V}, 0.40 \mathrm{~Hz}$ to $2.00 \mathrm{~Hz}$ ) in the $3 \mathrm{~min}$ signals (the first $3 \mathrm{~min}$ signals were continuously extracted from the 20 min signals). Significant differences $(\mathrm{p}<0.05)$ were found in frequency intervals III and V. The former exhibited the myogenic activity of smooth muscle inside the blood vessels in both $20 \mathrm{~min}$ and $3 \mathrm{~min}$ signal analyses; the latter showed hemodynamic oscillation caused by heart pumping. This finding agrees with the vascular pathological changes in Moyamoya disease. As a potential screening method for Moyamoya disease, the simple threshold method exhibited $73.5 \%$ accuracy.
\end{abstract}

Keywords: Moyamoya disease, near-infrared spectroscopy, cerebral oxygenation, wavelet transformation, screening

\section{Introduction}

Moyamoya disease, also known as "abnormal basicranial vascular network syndrome", is a chronic progressive cerebrovascular disease with unknown etiology. It is mainly characterized by spontaneous stenosis or occlusion at the terminal portions of the bilateral internal carotid artery and (or) the initial part of the anterior or middle cerebral artery. Cerebral angiography shows smoke-like abnormal basicranial vascular networks, from which the name of the disease has been derived [1].

The incidence of Moyamoya disease is high in East Asia. The prevalence and incidence rates in Japan were 3.16 and 0.35 per 100,000 population [1] in 1997, respectively, while the prevalence and

\footnotetext{
${ }^{1}$ Ying He, Pengjun Jiang and Shanshan Han contributed to this paper equally.

* Corresponding author: Tianxin Gao, School of Life Science, Beijing Institute of Technology, Beijing 100081, P.R. China. Tel.: 86-10-68912154; Fax: 86-10-68915956; E-mail: gtx@bit.edu.cn.
} 
annual incidence rates in Nanjing, China in 2010 were 3.92 and 0.43 per 100,000 population, respectively [2]. The onset age has two peaks: 5 and 40 years. As the disease progresses with stenosis, occlusion of the lesion basicranial vascular network and the compensatory generation of abnormal lateral capillary network, cerebral lesion of ischemia, and hemorrhage appear with the symptoms. The slow progress of Moyamoya disease and the lack of typical symptoms and signs make early detection difficult. Serious damage is usually incurred before diagnosis, leading to hemorrhagic or ischemic stroke. Thus, early detection and early clinical intervention can improve the prognosis of patients by delaying or even preventing disease progression and by mitigating brain function damage.

Digital subtraction angiography (DSA) is the gold standard of Moyamoya diagnosis. However, DSA is invasive, risky, and expensive. Thus, the use of DSA in screening is limited. Computed Tomography (CT), Magnetic Resonance Imaging (MRI), and Single Photon Emission Computed Tomography (SPECT) can be used to detect the stenosis of blood vessels as well as the altered hemodynamics of patients with Moyamoya disease. However, these methods are costly and lengthy, which make them impossible to be used in screening. A rapid, accurate, noninvasive method is needed in Moyamoya diagnosis and treatment.

Aside from the imaging methods previously mentioned, dynamic properties can also reflect the condition of cerebral blood vessels. Transcranial Doppler (TCD) and laser Doppler are two possible techniques. Laser Doppler can only detect superficial blood flow signals, whereas TCD is not always successful in detecting cerebral blood vessels [3]. Near-infrared spectroscopy (NIRS) is widely used in monitoring brain oxygenation during operation and brain oxygenation-related recognition study; thus, this technique can noninvasively detect the oxygenation condition of cerebral blood. The oscillations of cerebral blood oxygenation reflect the dynamic property of blood vessels indirectly as well as other aspects of cardiovascular regulation function, indicating a potential approach for the early diagnosis of Moyamoya disease through the dynamic property of blood vessels.

Using NIRS-based cerebral oxygenation signals, this study presents a screening method for Moyamoya disease by wavelet analysis of the oscillations in cerebral oxygenation. This method has been used for skin blood oscillations in patients with spinal cord injury and in healthy subjects [4]. It has also been used in spontaneous oscillations for age-related changes [5]. However, the present study is the first to use this method for Moyamoya disease screening.

\section{Materials and methods}

\subsection{Subjects}

A total of 17 healthy subjects ( 7 women, 10 men; average age $=37.4 \pm 11.3$ years) were recruited from Beijing Institute of Technology, and 17 subjects with Moyamoya disease (14 women, 3 men; average age $=40.1 \pm 11.2$ ) were recruited from Beijing Tian Tan Hospital, Capital Medical University. All subjects did not smoke or drink in the last $24 \mathrm{~h}$ before the measurement, which was carried out at least half an hour after any meal or activity. The measurement was performed while the subjects were awake with their eyes closed in comfortable supine position. The probe was placed on the forehead with the emitting end $1.5 \mathrm{~cm}$ apart from the forehead midline and $2 \mathrm{~cm}$ above the eyebrow, and the extension of the connection of the receiving and emitting ends intersected with the forehead midline in a 30 degree angle, avoiding the sagittal and frontal sinuses. The sensor was carefully fixed by a medical bandage on the forehead of each subject after cleaning with alcohol. The signals were continuously 
Table 1

Frequency interval division of the spontaneous oscillation of physiology signals

\begin{tabular}{|c|c|c|c|}
\hline Band & $\begin{array}{l}\text { Frequency interval } \\
(\mathrm{Hz})\end{array}$ & Band name & Physiology explanation \\
\hline I & $0.0095-0.02$ & Metabolic activity & $\begin{array}{l}\text { Hemodynamic change caused by the status switch of pre-capillary } \\
\text { sphincter }\end{array}$ \\
\hline II & $0.02-0.06$ & Neurogenic activity & Spontaneous activity of the sympathetic nervous system \\
\hline III & $0.06-0.15$ & Myogenic reaction & Myogenic activity of the smooth muscle inside the blood vessel \\
\hline IV & $0.15-0.40$ & Respiration & $\begin{array}{l}\text { Tidal change of blood flow dynamic parameters caused by respira- } \\
\text { tion }\end{array}$ \\
\hline $\mathrm{V}$ & $0.40-2.00$ & Heart pumping & Hemodynamic oscillations caused by heart pumping \\
\hline
\end{tabular}

monitored for $20 \mathrm{~min}$. Experiments were carried out in a quiet room with dim light at $26^{\circ} \mathrm{C}$ (room temperature). An informed consent was signed by each participant.

\subsection{NIRS measurements}

The NIRS measurement was performed using TSAH-100 [6] (Tsinghua University, China). According to the optical properties of human tissue in near-infrared wave band (700 nm to $900 \mathrm{~nm}$ ) [7], tissue oxygenation can be obtained.

The TSAH-100 sensor comprised two 760/850 nm double band LEDs as light sources and two PIN diodes as detectors. The two detectors were on the perpendicular bisector of the segment that joined the two sources. The distances between the two detectors and the perpendicular foot were $30 \mathrm{~mm}$ and $40 \mathrm{~mm}$, respectively [6]. The sampling rate of TSAH-100 was $20 \mathrm{~Hz}$. In NIR band, skin and skull are relatively transparent. Thus, the cerebral oxygenation parameters can be noninvasively detected by near-infrared (NIR) light [8]. Tissue oxygen parameters include the regional tissue oxygenation index (TOI), the difference between tissue oxygenated hemoglobin concentration and the value of its first point $\left(\Delta \mathrm{CHbO}_{2}\right)$, and the difference between tissue deoxygenated hemoglobin concentration and the value of its first point $(\triangle \mathrm{CHb})$.

\subsection{Wavelet and statistical analysis}

Wavelet is a time-frequency analysis method that is widely used in the spontaneous oscillations of the cardiovascular system. This method was used in blood flow oscillations in human skin and then in blood oxygenation signals. According to the existing studies, the frequency intervals were divided as shown in Table 1 [4,5,9-14].

Continuous signal length should be at least 10 times of the longest period in a time-frequency study. The shortest sampling time in low frequency analysis is $20 \mathrm{~min}$, including 10 periods of $0.0095 \mathrm{~Hz}$ signal. Li [5] obtained significant results by using 10 min signals (continuous signals with a length of $10 \mathrm{~min}$ ) in the analysis. This study presents a short-time screening method with a signal length of $3 \mathrm{~min}$ (the first $3 \mathrm{~min}$ signals were continuously extracted from the $20 \mathrm{~min}$ signals) according to the properties of Moyamoya disease. The average amplitude of the three frequency intervals (III, IV, and V) was calculated before the statistical analysis. The new method was compared with the traditional 20 min-signal-length and five-frequency-interval method. 


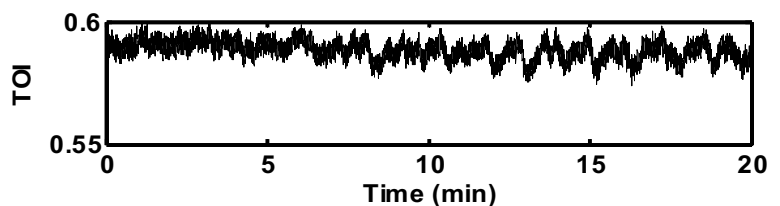

(a)

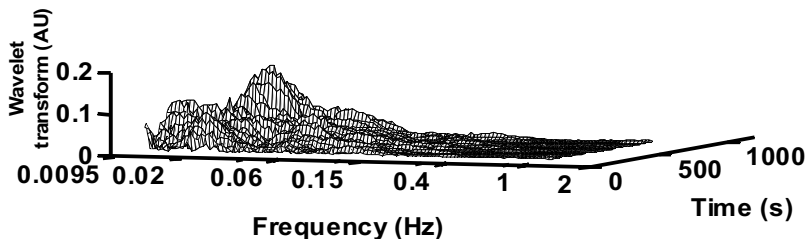

(b)

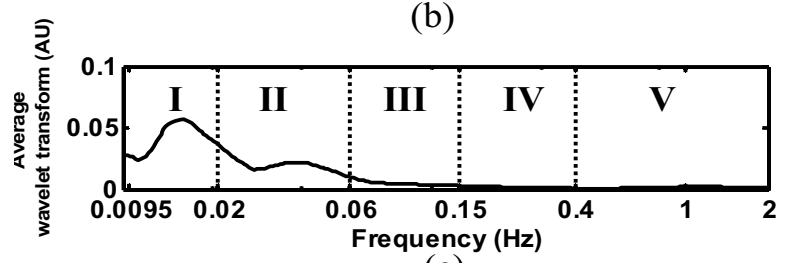

(c)

Fig. 1. Wavelet transformation result of TOI of a healthy subject. (a) The variation of original TOI measurement as a function of time $\mathrm{t}$ in $20 \mathrm{~min}$; (b) The time-frequency distribution of TOI after the wavelet transform; (c) Time averaging TOI as a function of frequency was divided into five frequency bands.

For the best time frequency resolution, Morlet wavelet was used in this study [13]. To minimize the boundary effect of the transformation, the first and the last one-sixth signals in the time-frequency domain after the wavelet transformation were deleted [14]. To average the 2D wavelet signal along the time axis, a 1D signal along the frequency axis was obtained. The average of the absolute amplitude of each frequency interval was calculated for statistical analysis [5,14].

One-way ANOVA was used to analyze the subjects' oxygenation parameters (TOI, $\Delta \mathrm{CHbO}_{2}$, and $\Delta \mathrm{CHb})$. Statistical significance was considered at $p<0.05$.

\section{Results}

\subsection{Wavelet analysis}

Figure 1 shows the wavelet transformation result of TOI of a healthy subject. Panel a shows the variation of the original TOI measurement as a function of time $t$. Panel b shows the time-frequency distribution of TOI after the wavelet transform. $x, y$, and $z$ axes represent the frequency, time, and signal amplitude after the wavelet transformation, respectively. By averaging the signal along the time direction, the distribution of TOI as a function of frequency was obtained and shown in panel c. The signals were divided into five frequency intervals, as performed in [4].

\subsection{Statistical analysis}

Figure 2 shows the results of the statistical analysis of TOI, $\Delta \mathrm{CHbO}_{2}$, and $\Delta \mathrm{CHb}$ between normal and Moyamoya subjects with a 20 min signal length. Figure 3 shows the results of a 3 min signal length. 
As shown in Table 2, significant differences were found in myogenic reaction and heart pumping intervals both in 20 min and 3 min signals. No significant difference was found in frequency interval I and II or in $\triangle \mathrm{CHb}$ signals.

A threshold method was used to separate Moyamoya patients from the normal people. In each band with significant difference, a threshold was chosen by average the mean observed values of the two groups (Moyamoya and normal subjects). The confusion matrix analysis was made and the results of the five classification methods were shown in Table 3. For clarity and easy comparison, results of each matrix were listed in one row.

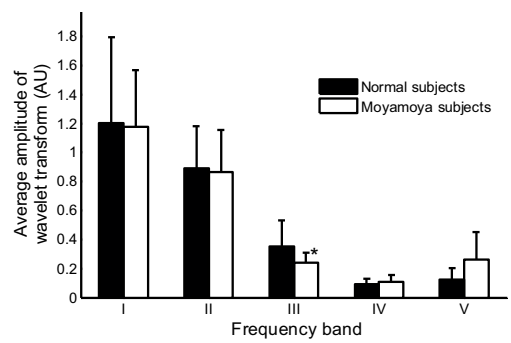

(a)

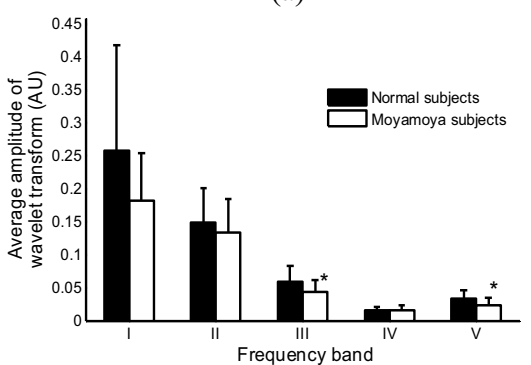

(b)

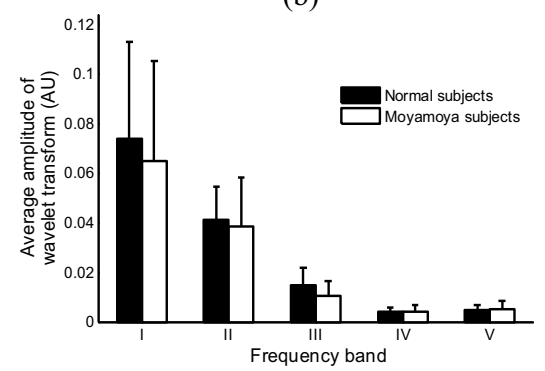

(c)

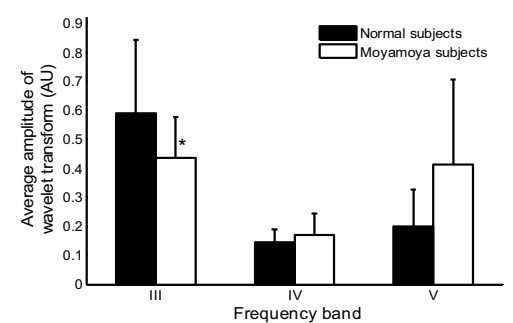

(a)

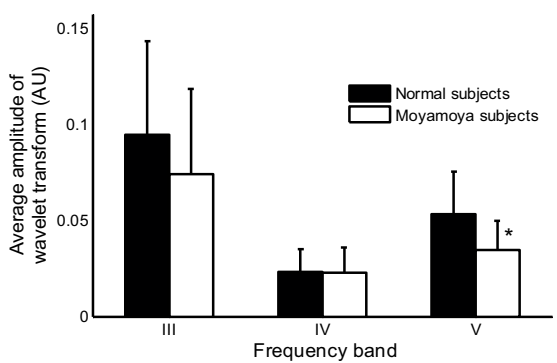

(b)

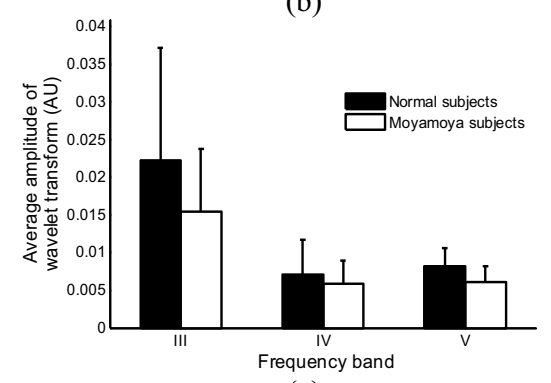

(c)

Fig. 2. Statistical result of (a) TOI, (b) $\Delta \mathrm{CHbO}_{2}$, and (c) $\triangle \mathrm{CHb}$ between normal and Moyamoya subjects with 20 min signal length. * represents significant difference.

Table 2

Calculated significant levels of 20 min signals and 3 min signals in the items with significant difference

\begin{tabular}{llll}
\hline Item & Frequency band & 20 min signals & 3 min signals \\
\hline TOI & III & 0.015 & 0.021 \\
$\Delta \mathrm{CHbO} 2$ & III & 0.042 & $-*$ \\
$\Delta \mathrm{CHbO} 2$ & $\mathrm{~V}$ & 0.031 & 0.016 \\
\hline Note: ${ }^{*} \mathrm{p}=0.166,>0.05$ & &
\end{tabular}


Table 3

Confusion matrix of different bands

\begin{tabular}{|c|c|c|c|c|c|c|c|c|c|c|c|c|}
\hline $\begin{array}{l}\text { Item - } \\
\text { Frequency } \\
\text { band }\end{array}$ & $\begin{array}{l}\text { Signal } \\
\text { length } \\
(\mathrm{min})\end{array}$ & A & B & $\mathrm{C}$ & $\mathrm{D}$ & $\begin{array}{l}\text { Sensibility } \\
\mathrm{A} /(\mathrm{A}+\mathrm{B})\end{array}$ & $\begin{array}{l}\text { Specificity } \\
D /(C+D)\end{array}$ & $\begin{array}{l}\text { False } \\
\text { positive } \\
\text { rate } \\
\mathrm{C} /(\mathrm{C}+\mathrm{D})\end{array}$ & $\begin{array}{l}\text { False } \\
\text { negative } \\
\text { rate } \\
\mathrm{B} /(\mathrm{A}+\mathrm{B})\end{array}$ & $\begin{array}{l}\text { Precision } \\
\mathrm{A} /(\mathrm{A}+\mathrm{C})\end{array}$ & $\begin{array}{l}\text { Negative } \\
\text { predictive } \\
\text { value } \\
\mathrm{D} /(\mathrm{B}+\mathrm{D})\end{array}$ & $\begin{array}{l}\text { Accuracy } \\
(\mathrm{A}+\mathrm{D}) / \\
(\mathrm{A}+\mathrm{B}+\mathrm{C}+\mathrm{D})\end{array}$ \\
\hline \multirow{2}{*}{ TOI - III } & 20 & 15 & 2 & 7 & 10 & $88.2 \%$ & $58.8 \%$ & $41.2 \%$ & $11.8 \%$ & $68.2 \%$ & $83.3 \%$ & $73.5 \%$ \\
\hline & 3 & 12 & 5 & 7 & 10 & $70.6 \%$ & $58.8 \%$ & $41.2 \%$ & $29.4 \%$ & $63.2 \%$ & $66.7 \%$ & $64.7 \%$ \\
\hline $\begin{array}{l}\Delta \mathrm{CHbO}_{2}- \\
\text { III }\end{array}$ & 20 & 11 & 6 & 6 & 11 & $64.7 \%$ & $64.7 \%$ & $35.3 \%$ & $35.3 \%$ & $64.7 \%$ & $64.7 \%$ & $64.7 \%$ \\
\hline$\Delta \mathrm{CHbO}_{2}-$ & 20 & 13 & 4 & 7 & 10 & $76.5 \%$ & $58.8 \%$ & $41.2 \%$ & $23.5 \%$ & $65.0 \%$ & $71.4 \%$ & $67.6 \%$ \\
\hline $\mathrm{V}$ & 3 & 14 & 3 & 7 & 10 & $82.4 \%$ & $58.8 \%$ & $41.2 \%$ & $17.6 \%$ & $66.7 \%$ & $76.9 \%$ & $70.6 \%$ \\
\hline
\end{tabular}

Note: A: true positive; B: false negative; C: false positive; D: true negative.

\section{Discussion}

The accuracy of the $20 \mathrm{~min}$ TOI-III band was $73.5 \%$, and that of the 3 min $\Delta \mathrm{CHbO}_{2}-\mathrm{V}$ band was $70.6 \%$. The results of the screening method for the long and short sampling times time were promising. Ultra-low signals may be removed to obtain useful information from the short-time signal when longtime monitoring is limited.

A large sample set is warranted to reduce the standard error and thus improve the accuracy of the results. The age-related threshold may be proposed to further classify the patients and healthy subjects. A weak relation was determined between the average amplitude of each frequency band and age in the healthy subjects. In addition, no definite conclusion can be drawn because of the limited sample size. Using the whole amplitude-frequency curve in Figure 1 (panel C) instead of the average amplitude of each frequency band as input and using a support vector machine can dramatically improve the accuracy of the results.

As shown in Table 3, the sensitivity was high ( $88.2 \%$ top), but the specificity (64.7\% top) was low. This result indicates that other factors might have contributed to the cerebral hemodynamic abnormality. Therefore, physiological reasons (e.g., low hemodynamic value in some healthy subjects) and other diseases (e.g., high blood pressure, carotid artery plaques) should be considered and studied before using this method as a screening method alone.

The reason behind the differences among the five bands remains under study. The spontaneous contract ability of the smooth muscle in the blood vessels of the Moyamoya patients was different from that of the healthy subjects. This difference may be attributed to the pathological alteration caused by Moyamoya disease. Histological analysis verified that the intracranial vascular lesions of Moyamoya disease exhibited intimal thickening, internal elastic lamina distortion, degeneration, and even rupture, with thinning or disappearance of the medial smooth muscle [15]. The heart pumping difference may be attributed to the lack of exercise among the patients. Further studies should be conducted to elucidate the relations between the wavelet analysis results and the pathophysiological markers of Moyamoya disease. Intraoperative and postoperative monitoring, as well as stage differentiation of the disease should also be conducted.

\section{Conclusion}

A significant difference was found in the spontaneous oscillation of cerebral oxygenation between 
Moyamoya and normal subjects. The most significant difference occurred in the TOI signal at the frequency interval of myogenic reaction (III: $0.06 \mathrm{~Hz}$ to $0.15 \mathrm{~Hz}$ ) and $\Delta \mathrm{CHbO}_{2}$ signal at heart pumping interval (V: $0.4 \mathrm{~Hz}$ to $2.0 \mathrm{~Hz}$ ).

The accuracy of the simple threshold method was $73.5 \%$ in the 20 min signals and $70.6 \%$ in the 3 min signals. Further improvement is necessary to make the wavelet analysis of cerebral NIRS signals a promising method in Moyamoya screening.

\section{Acknowledgement}

The work was supported by the basic research foundation of Beijing Institute of Technology (No. 20121642010).

\section{References}

[1] S. Kuroda and K. Houkin, Moyamoya disease: current concepts and future perspectives, Lancet Neurol. 7 (2008), $1056-1066$

[2] W. Miao, P.L. Zhao, Y.S. Zhang et al., Epidemiological and clinical features of Moyamoya disease in Nanjing, China, Clin. Neurol. Neurosurg. 112 (2010), 199-203.

[3] S. Moritz, P. Kasprzak, M. Arlt et al., Accuracy of cerebral monitoring in detecting cerebral ischemia during carotid endarterectomy: a comparison of transcranial Doppler sonography, near-infrared spectroscopy, stump pressure, and somatosensory evoked potentials, Anesthesiology 107 (2007), 563-569.

[4] Z. Li, J.Y. Leung, E.W. Tam et al., Wavelet analysis of skin blood oscillations in persons with spinal cord injury and able-bodied subjects, Archives of Physical Medicine and Rehabilitation 87 (2006), 1207-1212.

[5] Z. Li, M. Zhang, Q. Xin et al., Age-related changes in spontaneous oscillations assessed by wavelet transform of cerebral oxygenation and arterial blood pressure signals, Journal of Cerebral Blood Flow \& Metabolism 33 (2013), 692-699.

[6] H. Ding and Y. Teng, Non-invasively monitoring human tissue oxygenation using near infrared spectroscopy: independent innovations, Laser \& Optoelectronics Progress 44 (2007), 14-31.

[7] H. Obrig, C. Hirth, J.G. Junge-Hulsing et al., Cerebral oxygenation changes in response to motor stimulation, Journal of Applied Physiology 81 (1996), 1174-1183.

[8] E. Okada and D.T. Delpy, Near-infrared light propagation in an adult head model II: Effect of superficial tissue thickness on the sensitivity of the near-infrared spectroscopy signal, Applied Optics 42 (2003), 2915-2922.

[9] Y.K. Jan, B.D. Struck, R.D. Foreman et al., Wavelet analysis of sacral skin blood flow oscillations to assess soft tissue viability in older adults, Microvascular Research 78 (2009), 162-168.

[10] A. Humeau, A. Koïtka, P. Abraham et al., Time-frequency analysis of laser Doppler flowmetry signals recorded in response to a progressive pressure applied locally on anaesthetized healthy rats, Physics in Medicine and Biology 49 (2004), 843-857.

[11] T. Söderström, A. Stefanovska, M. Veber et al., Involvement of sympathetic nerve activity in skin blood flow oscillations in humans, American Journal of Physiology-Heart and Circulatory Physiology 284 (2003), H1638-H1646.

[12] A.B. Rowley, S.J. Payne, I. Tachtsidis et al., Synchronization between arterial blood pressure and cerebral oxyhaemoglobin concentration investigated by wavelet cross-correlation, Physiological Measurement 28 (2007), 161-173.

[13] A. Stefanovska, M. Bracic and H.D. Kvernmo, Wavelet analysis of oscillations in the peripheral blood circulation measured by laser Doppler technique, IEEE Transactions on Biomedical Engineering 4 (1999), 1230-1239.

[14] M. Bračič and A. Stefanovska, Wavelet-based analysis of human blood-flow dynamics, Bulletin of Mathematical biology 60 (1998), 919-935.

[15] C.A. David and E. Nottmeier, Intracranial occlusive disease, in: Youmans Neurological Surgery, H.R. Winn, ed., People's Medical Publishing House, Beijing, 2009, pp. 1362-1367. 